\title{
LA EQUIDAD Y LA SALUD EN EL TRABAJO: EXPLORANDO UN ÁREA EMERGENTE
}

\author{
INEQUITY AND HEALTH IN THE WORKPLACE: EXPLORING AN EMERGENT AREA
}

Luis Haro-García ${ }^{1, a}$, Guadalupe Aguilar-Madrid ${ }^{2, b}$, Cuauhtémoc A. Juárez-Pérez²,c, Sara D. Aguilar-Rodríguez ${ }^{3, d}$, Guillermo Flores-Carbajal ${ }^{3, e}$, Enrique Gea-Izquierdo ${ }^{4,5, f}$, Francisco R. Sánchez-Román ${ }^{2, a}$

\author{
Facultad de Medicina, Universidad Nacional Autónoma de México. México D.F, México \\ Instituto Mexicano del Seguro Social, México D.F, México. \\ Universidad Autónoma de la Ciudad de México. México D.F, México. \\ Dirección de Investigación e Innovación, Facultad de Salud y Seguridad Ocupacional, Universidad Internacional-SEK. Quito, Ecuador. \\ Cátedra de Seguriad y Salud en el Trabajo, Universidad de Málaga. Málaga, España. \\ Doctor en ciencias; ${ }^{b}$ doctora en ciencias; ${ }^{c}$ maestro en ciencias ambientales; ${ }^{\circledR}$ médico cirujano; ${ }^{e}$ médico especialista en psicoanálisis y formación de recursos \\ para la salud; ‘ biólogo doctor en ciencias biológicas, máster en Medicina preventiva y salud pública, máster en seguridad y salud en el trabajo \\ Recibido: 20-09-2013; Aprobado: 16-10-13
}

\begin{abstract}
RESUMEN
El trabajo, bajo condiciones de empleo justo y decente, reduce las inequidades en salud; sin embargo, en la actualidad parece prevalecer la obtención y desempeño de este sin los atributos referidos, con exposición tanto a accidentes como a padecer enfermedades causadas por diversos agentes, y a riesgos ergonómicos y psicosociales presentes en el trabajo, y los relacionados con el modo de trabajar impuesto por el wildmarket y sin prestaciones en materia de seguridad social. Las políticas que en salud y seguridad en el trabajo deberían instituir los países miembros de la Organización Internacional del Trabajo (OIT) acortarían las inequidades mencionadas, no obstante, el Estado, quien garantizaría esas políticas, parece haberse convertido en intermediador a favor de los grandes corporativos. Es fundamental que se definan y fortalezcan acciones para constituir empleos con condiciones apropiadas y decorosas, a fin de generar equidad, igualdad y bienestar social.
\end{abstract}

Palabras clave: Inequidad en salud; Salud ocupacional; Equidad; Bienestar social (fuente: DeCS BIREME).

\begin{abstract}
Work, under fair employment and decent work, reduces inequities in health. Nowadays it seems, however, that obtaining and carrying out a job and worker performance take precedence over the aforementioned attributes. Workers are not only exposed to accidents, diseases caused by various agents, ergonomic and psychosocial risks but also affected by work modes imposed by the "wildmarket", such as the lack of social security benefits. Member countries of the International Labour Organization (ILO) should institute occupational health and safety policies in order to reduce the above mentioned inequities. Nonetheless, governments, which would guarantee such policies, seem to have become intermediaries in favor of large corporations. It is essential to define and strengthen actions that create jobsin decent and appropriate conditions with a view to generating equity, equality, and social well-being.
\end{abstract}

Key words: Health inequalities; Occupational health; Equity; Social welfare (source: MeSH NLM).

\section{INEQUIDAD Y TRABAJO}

Las inequidades en materia de salud no son cosa nueva $y$, aunque al trabajo se le han adjudicado cualidades como el de promover la salud física y mental de los individuos, es indudable que también su práctica se ha asociado con la aparición de efectos adversos específicos para la salud, los cuales originan que se reduzcan las posibilidades de sostenibilidad y de obtener beneficios en el retiro ${ }^{(1,2)}$.

Quien no posee un trabajo está en mayores probabilidades de dañar su salud y mermar seriamente la de quienes dependen económicamente de él; ello motiva la aparición de la primera inequidad en materia de salud ocupacional: la de aquél quien posee un trabajo en condiciones justas y decente con el que no tiene posibilidades de obtener y desempeñar alguno con esos atributos ${ }^{(1,2)}$.

Visto de otra manera, se puede advertir la presencia de un gradiente de salud en la población considerada, desde el punto de vista social, como clase trabajadora, a quienes se les califica como poseedores de condiciones de salud menos favorables frente a quienes se encuentran jerárquicamente por arriba, pero que están en mejores

Citar como: Haro-García L, Aguilar-Madrid G, Juárez-Pérez CA, Aguilar-Rodríguez SD, Flores-Carbajal G, Gea-Izquierdo E, et al. La equidad y la salud en el trabajo: explorando un área emergente. Rev Peru Med Exp Salud Publica. 2013;30(4):671-5. 
condiciones cuando son asalariados(1). En este sentido debe destacarse que la Comisión de los Determinantes Sociales para la Salud de la Organización Mundial de la Salud (OMS) ha establecido que el trabajo, en particular, bajo condiciones de empleo apropiadas y decorosas puede, de hecho, complementar las necesidades que permiten reducir las inequidades en salud en su más amplio concepto ${ }^{(2)}$.

Bajo el escenario que se señala, y tal como se menciona en el análisis denominado Employment Conditions and Health Inequalities de esa misma comisión, centrar al trabajo en la configuración de la clase social es pieza fundamental para entender el complejo mundo de inequidades que existen en y por esta actividad, y la otra, las condiciones específicas que la rodean, lo que según algunos autores, posibilitan la construcción de una visión más completa e integral del problema, apoyado en que, por ejemplo, la frecuencia de desnutrición, bajo peso al nacer, el surgimiento de enfermedades mentales o cardiovasculares, se observan más entre aquellos individuos que se catalogan como desempleados ${ }^{(3,4)}$.

En cuanto a los que ocupan y desempeñan un puesto de trabajo, al menos hasta el año 2012, se debía hacer referencia a una población de más de tres mil millones de individuos que efectivamente laboraban y que al mismo tiempo se exponían en condiciones desiguales a diversos grados de riesgos ocupacionales, de tal modo que se ha llegado a estimar que a nivel mundial alrededor de 700000 de ellos se lesionan por día y 350000 mueren anualmente por accidentes fatales, lo que significa que casi mil fallecen diariamente por esta causa ${ }^{(3,4)}$.

Lo anterior no toma en cuenta las enfermedades causadas por el trabajo, las cuales son comúnmente subdiagnosticadas o perversamente subregistradas, estas se deben a la exposición ocupacional crónica a metales pesados; a polvos como el asbesto, el carbón y el sílice; a plaguicidas agroquímicos, disolventes orgánicos, y múltiples sustancias en el ambiente de trabajo reconocidas como cancerígenos o disrruptores endócrinos; también están las enfermedades causadas por agentes físicos como el ruido industrial, y los de orden biológico y disergonómicos; adicionalmente están las que poseen características psicosociales en la organización laboral y que se traducen como mobbing, karoshi, burnout o estrés laboral en la población trabajadora ${ }^{(5-14)}$.

El escenario anterior debe de incluir lo relacionado con el ejercicio de nuevos modos de trabajar impuestos por el wildmarket o "mercado salvaje", caracterizado por jornadas extenuantes de trabajo de más de ocho horas; imposición y abuso en la ejecución de dobles o triples jornadas de labor; el trabajo informal; las desigualdades en cuanto a género y trabajo; trabajo infantil, y otros, aunado a la ausencia de beneficios de prestaciones en materia de seguridad social que se ha percibido en todo el orbe en el último cuarto del siglo $\mathrm{XX}$ y que coloca a la clase trabajadora en condiciones similares de trabajo precario, de alta vulnerabilidad y explotación que existían a fines del siglo XIX ${ }^{(15,16)}$.

Por otro lado, la globalización, la aparición de nuevas tecnologías y la exigencia con tintes de usura que los bloques y corporativos financieros ejercen sobre buena parte de los países de todo el mundo para que den cumplimiento a compromisos de orden económico a costa de los derechos de los trabajadores, han provocado cambios profundos tanto en las condiciones de trabajo, con recortes o pérdida de esos mismos derechos ${ }^{(4)}$.

Lo anterior se acompaña de características que sirven para segregar al momento que se contrata a un trabajador, la aceptación o no de una persona se establece por pertenecer a determinado sexo, edad, grupo étnico o social; incluso si existe obesidad; que no encuadre con un patrón particular de belleza; que exista sospecha de gestación; que se marquen preferencias por emplear a parientes o por privilegiar lazos de amistad aunque no se posean habilidades, aptitudes, experiencia previa, o demostrar profesionalización formal en algunas destrezas que debe cubrir el aspirante o candidato a un puesto de trabajo ${ }^{(1,16-18)}$.

\section{DISTRIBUCIÓN DE LA INEQUIDAD EN LA SALUD DE LOS TRABAJADORES}

Las inequidades en salud, y entre ellas las relacionadas con el trabajo, no se deben visualizar como "naturales" o como una característica inmutable de la sociedad. De hecho, buena parte de la distribución geográfica de estas inequidades no es dada por las posibilidades de acceso a los sistemas de atención a la salud, aunque ello cumpla un importante papel al momento de evaluarlas ${ }^{(4)}$

Las inequidades en salud surgen sistemáticamente de las inequidades sociales, y esas han sido posible establecerlas a partir de lo que se ha denominado como patrón de variación en la distribución de las inequidades que se ha dado a través del tiempo entre países o regiones, y dentro de estos mismos, y el cual responde a cambios políticos, económicos, sociales y culturales que se dan tanto de forma interna como externa. Un ejemplo de ello son las observaciones 
que se realizaron en población de Inglaterra y país de Gales, donde se identificó que la tasa de mortalidad en hombres de 15 a 64 años de edad de los vecindarios más económicamente deprimidos fue de 2,5 más veces que en los individuos de vecindarios menos deprimidos. En adición, se identificó una diferencia de hasta 17 años en la expectativa de vida libre de discapacidad entre trabajadores que poseen mejores ingresos en comparación a aquellos con más bajos ingresos ${ }^{(19-21)}$.

Pese a esos señalamientos tan elocuentes, y en referencia a los países escandinavos en quienes se llegó a pensar que presentaba las menores inequidades en salud, incluidas las relacionadas con el empleo y el trabajo en razón de su provisión generosa y universal de bienestar, no pudo demostrarse que ello se cumpliera del todo en esos países y que más bien su situación en materia de inequidades es intermedia; sugiriendo dicha observación que el patrón no siempre parece comportarse de manera lineal en cuanto a que las inequidades en salud son en función del ingreso de un país o región. Tal podría ser el caso de Cuba, que pese a sus rezagos económicos, se ha señalado como el país mejor posesionado en cuanto a menores inequidades en salud en comparación con toda Latinoamérica y el Caribe, por arriba de Argentina, Uruguay, Chile, México y Brasil, en ese orden ${ }^{(4,22)}$.

Analizado desde otra perspectiva, estimaciones del Banco Mundial revelaron que China e India, países de economía emergente, el riesgo de lesiones fatales y no fatales a causa del trabajo era 2,5 veces más alta que la observada en Europa y Estados Unidos; este mismo riesgo se eleva al doble en países del África subsahariana ${ }^{(4)}$.

En términos de enfermedades de trabajo, su distribución parece, lamentablemente, tener un aspecto más uniforme ya que se presentan altos índices de subregistro de hasta más del $60 \%$ de casos, aun en países tecnológicamente avanzados como los Estados Unidos y países centro europeos pese a que Alemania ha sido señalado como el país que posee las menores inequidades ${ }^{(23)}$

Por lo que toca a los trabajadores en América Latina y el Caribe, se ha señalado que solamente el $30 \%$ de aquellos que pertenecen al sector formal reciben algún tipo de atención en salud ocupacional, que por lo general estuvo dirigida al tratamiento y no a la prevención o a la promoción. Las tasas de accidentes mortales en el trabajo fueron 2,5 veces mayores en América Latina y el Caribe que en los Estados Unidos y el Canadá, y desde 1992, las de los trabajadores de la construcción de origen latinoamericano han sido marcadamente mayores que las de los demás trabajadores. Además, $44 \%$ de los trabajadores de esta región perciben salarios debajo de la línea de pobreza y 19,4\% tienen salarios debajo de la línea de pobreza extrema. La Organización Panamericana de la Salud (OPS) estima que en Honduras y Nicaragua existen entre 8 y $12 \%$ de niños y de jóvenes menores de 18 años que trabajan o viven en las calles. Por otro lado, la Organización Internacional del Trabajo (OIT), señala que entre 1992 y 2002 la economía informal de América Latina creció de 42,8 a $46,5 \%{ }^{(24)}$.

En cuanto a la frecuencia de enfermedades ocupacionales en las Américas, cuando se formuló el Plan Regional de la Organización Panamericana de la Salud (OPS), a finales del decenio de 1990, se estimó que en América Latina y el Caribe solo se notificaban entre 1 y $4 \%$ de los casos. Pese a que se propuso fortalecer las estrategias para el diagnóstico y reconocimiento de estas enfermedades en toda la región, los resultados no son muy prometedores. En México, por ejemplo, entre 1992 y 2002, el Instituto Mexicano del Seguro Social (IMSS) registró 5212372 riesgos de trabajo, de los cuales menos del $1 \%$ fueron catalogados como enfermedades de trabajo ${ }^{(24)}$.

\section{PERSPECTIVAS DE EQUIDAD EN EL TRABAJO}

No existen dudas de que el seguimiento a las políticas que en salud y seguridad en el trabajo que deberían dar los países miembros de la OIT acortarían las inequidades mencionadas. La salvaguarda del impacto multipolar de los ajustes al empleo bajo condiciones precarias, con alta flexibilización y sin seguridad laboral, aplicadas a la fuerza de trabajo con contrato mediado por agencias, outsourcing, o de forma temporal, debería ser insignia contra esa actualidad globalizada, representada por la prosperidad de unos cuantos arropados en crisis financieras o manejadas a su propia conveniencia $(1,3,6,7,17,18,22)$.

En ese entramado, el Estado, quien debería ser la supraestructura que garantiza las políticas en materia de salud y trabajo, parece haberse convertido en intermediador de los grandes corporativos, más aun, es notoria la ausencia en las agendas nacionales de temas relacionados con la salud de los trabajadores $(5,16,25)$.

La traducción más exacta de ese juicio ha sido la pérdida de fuentes de trabajo, en mayor proporción entre hombres jóvenes, tal y como sucedió de manera reciente en tres países pre-bálticos, España e Irlanda con respecto al resto de Europa, hasta en seis puntos 
porcentuales, principalmente en el sector económico de la construcción y de la manufactura; ello no quiso decir que a las mujeres hayan corrido con mejor suerte, ya que por lo general fueron a las que más rápido se les despidió de las empresas, a quien de manera anticipada se les redujo el salario o se le discriminó con mayor frecuencia ${ }^{(4,18)}$.

Por otro lado, aquellos trabajadores que pertenecían al sector público tampoco han quedado exonerados de los recortes de todo tipo, con serios daños en sus derechos laborales cuando están en activo y los que correspondiesen en el en retiro, algo que solo era común que aconteciera a trabajadores al servicio del sector privado ${ }^{(16)}$.

\section{CONCLUSIÓN}

Es fundamental que a corto, medio y largo plazo se definan con mayor atención las acciones a tomar por los pueblos del mundo y sus gobiernos, o al menos la mayor parte de las regiones de este sobre la crisis macroeconómica por la que están atravesando el planeta, ya que precisamente esa insistente postura globalizadora hace que también sea de orden laboral, ambiental, alimentaria, energética y política, en el que un relevante indicador del dominio y control de estas sea el trabajo en condiciones de empleo justo $\mathrm{y}$ decente, con su poder para generar igualdad y bienestar social ${ }^{(4,25-28)}$.

De acuerdo a como se presenta el panorama, en términos de equidad y salud en el trabajo, el reforzamiento de este último como derecho individual y colectivo, es la opción a seguir pese al escenario actual claramente desfavorecedor, pero con la convicción de que ello no será permanente, lo que alentará que el derecho del trabajo dejará de ser lo que es para constituirse en lo que debe ser: derecho reivindicador de la especie humana $(25,26)$.

Contribuciones de autoría: LHG participó de la concepción y diseño del artículo; GAM, CAJP y FRSR contribuyeron en la recolección de material y literatura relacionadas al tema, en la redacción del artículo y en su revisión crítica; SDAR y GFC participaron en la revisión crítica del artículo, abundaron en su concepción y aportaron datos; EGF participó en la revisión crítica del artículo, en su estructura y contribuyó en su redacción. Todos los autores aprobaron la versión final.

Fuentes de financiamiento: autofinanciado.

Conflictos de interés: los autores declaran no tener conflictos de interés.

\section{REFERENCIAS BIBLIOGRÁFICAS}

1. Marmot M, Bell R. Challenging health inequalities--implications for the workplace. Occup Med (Lond). 2010;60(3):162-4. doi: 10.1093/ occmed/kqq008.

2. World Health Organization, Commission on Social Determinants of Health. Closing the gap in a generation: health equity through action on the social determinants of health. Final Report. [Internet]. Geneva: World Health Organization; 2008 [citado el 1 de agosto del 2013]. Disponible en: http://whqlibdoc.who.int/publications/2008/9789241563703_eng.pdf

3. World Health Organization, Commission on Social Determinants of Health. Employment conditions and health inequalities. Final Report. [Internet]. Geneva: World Health Organization; 2007 [citado el 1 de agosto del 2013]. Disponible en: http://www.who.int/social_ determinants/resources/articles/ emconet_who_report.pdf

4. Tamez-González S, Pérez-Domínguez J.La sociedad del riesgo y las inequidades en la salud de los trabajadores. Rey Salud Publica. 2012;14 Supl 1:43-55.
5. Sánchez-Román FR, Juárez-Pérez CA, Aguilar-Madrid G, Haro-García L, Borja-Aburto VH, Claudio L. Occupational health in Mexico. Int J Occup Environ Health. 2006; 12(4):346-54.

6. Noone P. Social determinants of health. Occup Med (Lond). 2009;59(3):209.

7. Haro-García L, Sánchez-Román RF, Juárez-Pérez CA, Larios-Díaz E. Justificaciones médicas de la jornada laboral máxima de ocho horas. Rev Med Inst Mex Seguro Soc. 2007;45(2):191 7.

8. Shields M. Long working hours and health. Health Rep. 1999;11(2):33-48.

9. McNeely EM. Who's counting anyway? The problem with occupational safety and health statistics. J Occup Med. 1991;33(10):1071-5.

10. Ke DS. Overwork, stroke, and karoshideath from overwork. Acta Neurol Taiwan. 2012;21(2):54-9.

11. Cabrera-Gutiérrez LS, López-Rojas P, Salinas-Tovar S, Ochoa-Tirado JG, Marìn-Cotoñieto IA, Haro-Garcia L. Burnout syndrome among Mexican hospital nursery staff. Rev Med Inst Mex Seg Soc. 2005;43(1):11-5.

12. Rondinone BM, Persechino B, Castaldi T, Valenti A, Ferrante P, Ronchetti M, et al. Work-related stress risk assessment in Italy: the validation study of health safety and execute indicator tool. G Ital Med Lav Ergon. 2012;34(4):392-9.

13. Toral-Villanueva R, Aguilar-Madrid G, Juárez-Pérez CA. Burnout and patient care in junior doctors in Mexico City. Occup Med (London). 2009;59(1):813. doi: $10.1093 /$ occmed/kqn122.

14. García-Lechuga Y, Aguilar-Madrid G, Sánchez-Román FR, Juárez-Pérez CA, Haro-García L. Prevalencia de síndrome de fatiga crónica en trabajadores de una fábrica mexicana de cierres. Revista Médica de Risaralda. 2012;18(1):29-35.

15. Dudarev AA, Odland JØ. Occupational health and health care in Russia and Russian Arctic: 1980-2010. 2013;72:20456. doi: 10.3402/ijch. v72i0.20456.

16. Sánchez-Román FR, Pérez-Padilla A, Sánchez-Vizcaíno PM, Ortega- 
Escudero MT, Pérez-Martínez P, HaroGarcía L. Reflexiones en torno a los 40 años de la medicina del trabajo en México. Rev Med Inst Mex Seguro Soc. 2007;45(4):403-12.

17. Jacson T. Prosperity without growth? The transition to a sustainable economy. London: The Sustainable Development Commission; 2009.

18. Vaughan-Whitehead D. Work inequalities in the crisis. Evidence from Europe. Edward Elgar Publishing; 2011.

19. Marmot MG, Smith GD, Stansfeld S, Patel C, North F, Head J, et al. Health inequalities among British civil servants: the Whitehall II study. Lancet. 1991 Jun 8;337(8754):1387-93.

20. North MF, Syme L, Feeney A, Shipley M, Marmot M. Psychosocial work environment and sickness absence among British civil servants: the Whitehall II study. Am J Public Health. 1996;86(3):332-40.
21. Marmot M. Fair society, healthy lives: strategic review of health inequalities in England Post 2010. London: Marmot Review; 2010.

22. Cardona D, Acosta LD, Bertone CL. Inequidades en salud entre países de Latinoamérica y el Caribe (20052010). Gac Sanit. 2013;27(4):292-7.

23. Pransky G, Snyder T, Dembe A, Himmelstein J. Under-reporting of work-related disorders in the workplace: a case study and review of the literature. Ergonomics. 1999;42(1):171-82.

24. Organización Panamericana de la Salud, Oficina Sanitaria Panamericana, Oficina Regional de la Organización Mundial de la Salud. Salud en las Américas. Publicación científica y técnica No. 622. Volumen I-Regional. Washington, DC: OPS; 2007.

25. Marx K. Libro Primero: El proceso de producción del capital. Capítulo XII: División del Trabajo y Manufactura.
En: Marx K. El Capital. México, DF: Grupo Editorial; 2004.

26. Delgado-Moya R. Filosofía del derecho del trabajo. México, DF: Editorial Pac; 1993.

27. Lee KM, Yang JS, Kim G, Lee J, Goh KI, Kim IM. Impact of the topology of global macroeconomic network on the spreading of economic crises. PLoS One. 2011 Mar 31;6(3):e18443. doi: 10.1371/journal.pone.0018443.

28. Deaton A. The financial crisis and the well-being of Americans. Oxf Econ Pap. 2012;64(1):1-26.

\section{Correspondencia: Luis Haro Garcia}

Dirección: Edificio C, Unidad de Investigación en Salud en el Trabajo, Primer Piso, Centro Médico Nacional "Siglo XXI", Instituto Mexicano del Seguro Social, Av. Cuauhtémoc 330, Colonia Doctores, Delegación Cuaubtémoc, CP: 06725, México, Distrito Federal, México. Correo electrónico:luisharo2@hotmail.com

\section{Consulte la versión electrónica de la Revista Peruana de Medicina Experimental y Salud Pública en} www.pubmed.gov

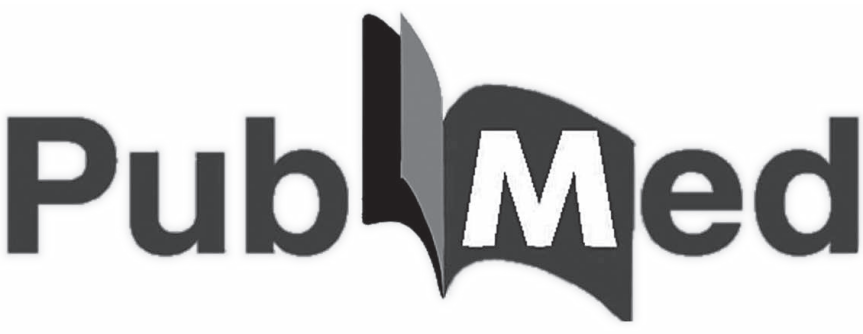

